\title{
Acknowledging the Unsung Role of the Cancer Registry in Rare Cancers
}

\author{
Jung-Myun Kwak \\ Division of Colorectal Surgery, Department of Surgery, Korea University Anam Hospital, Korea University College of Medicine, Seoul, Korea
}

\section{See Articles on Page 35-40}

Recording mortality statistics to understand causes of death dates back to at least the late 16th century. "Bills of mortality" for each parish of London were produced starting in November 1532 to monitor burials during outbreaks of the plague. In 1662, a London businessman, John Graunt, invented the science of medical epidemiology and statistics, using observational data to inform clinical practice [1]. He provided the first recorded descriptions of the patterns of different causes of death. Around the same time, other observations about disease causation were described in areas such as occupational cancer and diseases caused by nutritional deficiencies. Bernardino Rammazzini noted the increased risk of breast cancer in nuns, and Percival Pott recognized the association between scrotal cancer and chimney sweeps. The story of how Captain James Cook prevented scurvy using fresh food and lemon juice is very well known [1].

By the 20th century, with antibiotics successfully addressing the majority of infectious diseases, the focus of epidemiology shifted to cancer [2]. The first modern registries for the study of cancer emerged in the early 1900s by individual physicians or as institutional projects in the United States and Europe. At the outset, observational data were collected to elucidate cancer causation, and epidemiologic studies generated population data identifying risk factors for cancers. Such information demonstrated the associations between environmental, dietary, hormonal, and other exogenous exposures and increased risk of cancer [3]. During the past

Correspondence to: Jung-Myun Kwak, M.D.

Division of Colorectal Surgery, Department of Surgery, Korea University Anam Hospital, Korea University College of Medicine, 73 Goryeodae-ro, Seongbuk-gu, Seoul 02841, Korea

Tel: +82-2-920-5323, Fax: +82-2-928-1631

E-mail:jmkwak@korea.ac.kr

ORCID: https://orcid.org/0000-0002-2181-4279

(C) 2020 The Korean Society of Coloproctology

This is an open-access article distributed under the terms of the Creative Commons Attribution NonCommercial License (https://creativecommons.org/licenses/by-nc/4.0) which permits unrestricted noncommercial use, distribution, and reproduction in any medium, provided the original work is properly cited. three decades, the advent of technologies to identify biological molecules has facilitated development of modern molecular/genetic epidemiology, which has enabled identification of genetic variants and their products as correlates of cancer risk [2].

Like the known main goals of traditional epidemiology, there are several goals of a cancer registry. First, the cause and risk of cancer need to be identified. Just as the ultimate goal of epidemiology is to reduce prevalence and mortality of a disease by providing reasonable evidence of how to prevent it, we need to identify the causes and risks of specific cancers and take preventive measures based on data from the cancer registry. Second, it is necessary to evaluate the incidence of cancer in the community and the cost to society. Armed with this information, health care services and facilities not only prepare and plan treatments, but also develop tailored training for health care professionals. Third, the registry should be used as a means of investigating and evaluating the efficacy of cancer prevention and treatment as well as health delivery systems and to evaluate how effectively health care services have prevented cancer and improved early diagnosis and survival.

Most resources and efforts have focused on cancers of high incidence, such as gastric, lung, colon, and breast cancer. Although classic epidemiologic study will undoubtedly continue contributing to cancer prevention in common cancers, a huge amount of biological, genetic, and clinical data has accumulated based on numerous basic and clinical studies to standardize diagnosis and treatment. With the evolving understanding of cancer biology, a new medical model called 'precision medicine,' which proposes customization of healthcare, is emerging.

Hidden from the limelight, there are many kinds of rare cancers, and their social burdens cannot be ignored. According to the rare cancer definition (incidence $<6 / 100,000 / y r$ ) of The Project Surveillance of Rare Cancers in Europe (RARECARE), 541,000 new diagnoses of rare cancers occur each year in Europe, accounting for $22 \%$ of all cancer diagnoses [4]. Rare cancers have a low incidence, and doctors lack associated expertise, which causes difficulties in clinical decision making for proper diagnosis and treatment. It is very difficult to recruit a sufficient number of such patients for clinical trials, limiting development and validation of ap- 
propriate treatment policies. Most rare cancers are difficult to centralize to specialized centers, which highlights the importance of collection of information such as annual incidence, survival, and prevalence of rare cancers through the national cancer registry.

The Korea Central Cancer Registry (KCCR), a nationwide, hospital-based cancer registry, was initiated by the Korean Ministry of Health and Welfare in 1980. In 1999, the KCCR expanded cancer registration to cover the entire Korean population under the Population-Based Regional Cancer Registry program [5]. This database represented $98.2 \%$ of the total cancer incidence in 2016, as determined by the Ajuki method [6]. Accurate and complete information obtained through the KCCR can be used to define and monitor cancer incidence, enable epidemiological studies, determine the direction of health care policy, and improve treatment of cancer patients. Its value will be especially notable in rare cancers where clinical studies are difficult. This information will help to better identify trends in specific cancers and to optimize medical resources to prevent and treat cancer.

Hur et al. [7] analyzed KCCR data for assessing the clinical features and survival of anal cancer, which is a rare cancer in Korea. Data from 3,615 patients during the past 20 years were retrieved and showed an annual increase in incidence of anal cancer from 552 cases in 1996-2000 to 1,062 cases in 2011-2015. The analyses showed improved survival over time, with an increase in 5-year relative survival rate from $39.7 \%$ in $1993-1995$ to $66.5 \%$ in $2011-$ 2015. The results illustrate significant trends in incidence and survival of Korean anal cancer. Although the study did not provide information on risk factors, it would be possible to interpret and explain the increase in incidence in terms of well-known risk factors, such as human papilloma virus infection, smoking, sexual practice, immunosuppression, and human immunodeficiency virus infection. Such information might be helpful in identifying social phenomena and developing preventive measures. Although the influence of treatment modalities on improvement in survival could not be evaluated, there is no doubt that the research will be an important reference in the study of anal cancer in Korea.

Data sources of big data in oncology are plentiful, containing demographic details, radiological data, tissue-based analysis including genetic expression, and data from patients and medical professionals. Nevertheless, cancer surveillance of basic statistics in a population-based cancer registry plays a key role in formulating cancer control plans, as well as in monitoring their success. This is most benefitted by rare cancers, however, data generated from the cancer registry must be integrated into clinical research in order to play an expanded role in such rare cancers.

\section{CONFLICT OF INTEREST}

No potential conflict of interest to this article is reported.

\section{ACKNOWLEDGMENTS}

I thank Hyunmi Park for her kind assistance in proofreading the manuscript.

\section{REFERENCES}

1. Potter JD. Epidemiology informing clinical practice: from bills of mortality to population laboratories. Nat Clin Pract Oncol 2005; 2:625-34.

2. Greenwald P, Dunn BK. Landmarks in the history of cancer epidemiology. Cancer Res 2009;69:2151-62.

3. White MC, Babcock F, Hayes NS, Mariotto AB, Wong FL, Kohler $\mathrm{BA}$, et al. The history and use of cancer registry data by public health cancer control programs in the United States. Cancer 2017; 123 Suppl 24:4969-76.

4. Gatta G, van der Zwan JM, Casali PG, Siesling S, Dei Tos AP, Kunkler I, et al. Rare cancers are not so rare: the rare cancer burden in Europe. Eur J Cancer 2011;47:2493-511.

5. Shin HR, Won YJ, Jung KW, Kong HJ, Yim SH, Lee JK, et al. Nationwide cancer incidence in Korea, 1999 2001; first result using the national cancer incidence database. Cancer Res Treat 2005;37: 325-31.

6. Jung KW, Won YJ, Kong HJ, Lee ES. Cancer statistics in Korea: incidence, mortality, survival, and prevalence in 2016. Cancer Res Treat 2019;51:417-30.

7. Hur H, Jung KW, Kim BW, Oh CM, Won YJ, Oh JH, et al. Longterm oncologic outcome and its relevant factors in anal cancer in korea: a nationwide data analysis. Ann Coloproctol 2020;36:3540. 